\title{
Correction to: China's health assistance to Africa: opportunism or altruism?
}

Shuang Lin ${ }^{1}$, Liangmin Gao ${ }^{2}$, Melissa Reyes ${ }^{3}$, Feng Cheng ${ }^{4,5}$, Joan Kaufman ${ }^{6,7,4}$ and Wafaa M. El-Sadr ${ }^{8,3^{*}}$

\section{Correction}

Please note that following publication of the original article [1], one of the authors has flagged that the abbreviations section lists "BRIC" as "Britain, Russia, India and China".

However, in accordance with reference '38' [2], in relation to which 'BRIC' is referred, the correct meaning of BRIC is in fact: "Brazil, Russia, India and China".

\begin{abstract}
Author details
${ }^{1}$ School of International and Public Affairs, Columbia University, New York, USA. ${ }^{2}$ School of Social Sciences, Tsinghua University, Beijing, People's Republic of China. ${ }^{3}$ Mailman School of Public Health, Columbia University, New York, USA. ${ }^{4}$ Research Center for Public Health, Tsinghua University, Beijing, People's Republic of China. ${ }^{5}$ Center for Global Health and Infectious Diseases, Tsinghua University School of Medicine, Beijing, People's Republic of China. ${ }^{6}$ Schwarzman Scholars Program, Stephen A. Schwarzman Education Foundation, New York, USA. ${ }^{7}$ Global Health and Social Medicine, Harvard Medical School, Boston, USA. ${ }^{8}$ ICAP at Columbia University, New York, USA.
\end{abstract}

Published online: 01 October 2018

\section{References}

1. Lin et al. China's health assistance to Africa: opportunism or altruism? 2016 12:83 DOI: https://doi.org/10.1186/s12992-016-0217-1.

2. Jakovljevic et al.. The key role of the leading emerging BRIC markets in the future of global health care. 2014 15:3 DOI https://doi.org/10.2478/sjecr2014-0018.

\footnotetext{
*Correspondence: wme1@columbia.edu

${ }^{8}$ ICAP at Columbia University, New York, USA

${ }^{3}$ Mailman School of Public Health, Columbia University, New York, USA

Full list of author information is available at the end of the article
} 